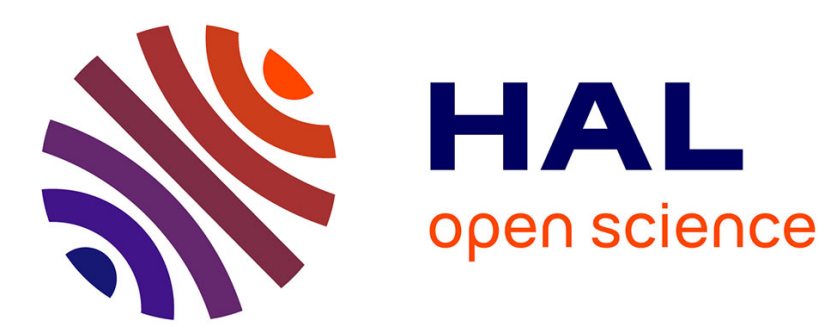

\title{
Exactly Solvable Stochastic Processes for Traffic Modelling
}

Maxim Samsonov, Cyril Furtlehner, Jean-Marc Lasgouttes

\section{To cite this version:}

Maxim Samsonov, Cyril Furtlehner, Jean-Marc Lasgouttes. Exactly Solvable Stochastic Processes for Traffic Modelling. [Research Report] RR-7278, INRIA. 2010, pp.26. inria-00492430

\section{HAL Id: inria-00492430 \\ https://hal.inria.fr/inria-00492430}

Submitted on 15 Jun 2010

HAL is a multi-disciplinary open access archive for the deposit and dissemination of scientific research documents, whether they are published or not. The documents may come from teaching and research institutions in France or abroad, or from public or private research centers.
L'archive ouverte pluridisciplinaire HAL, est destinée au dépôt et à la diffusion de documents scientifiques de niveau recherche, publiés ou non, émanant des établissements d'enseignement et de recherche français ou étrangers, des laboratoires publics ou privés. 


\title{
I N R I A
}

INSTITUT NATIONAL DE RECHERCHE EN INFORMATIQUE ET EN AUTOMATIQUE

\section{Exactly Solvable Stochastic Processes for Traffic Modelling}

\author{
Maxim Samsonov — Cyril Furtlehner — Jean-Marc Lasgouttes
}

$\mathbf{N}^{\circ} \mathbf{7 2 7 8}$

Mai 2010

Domaine 1

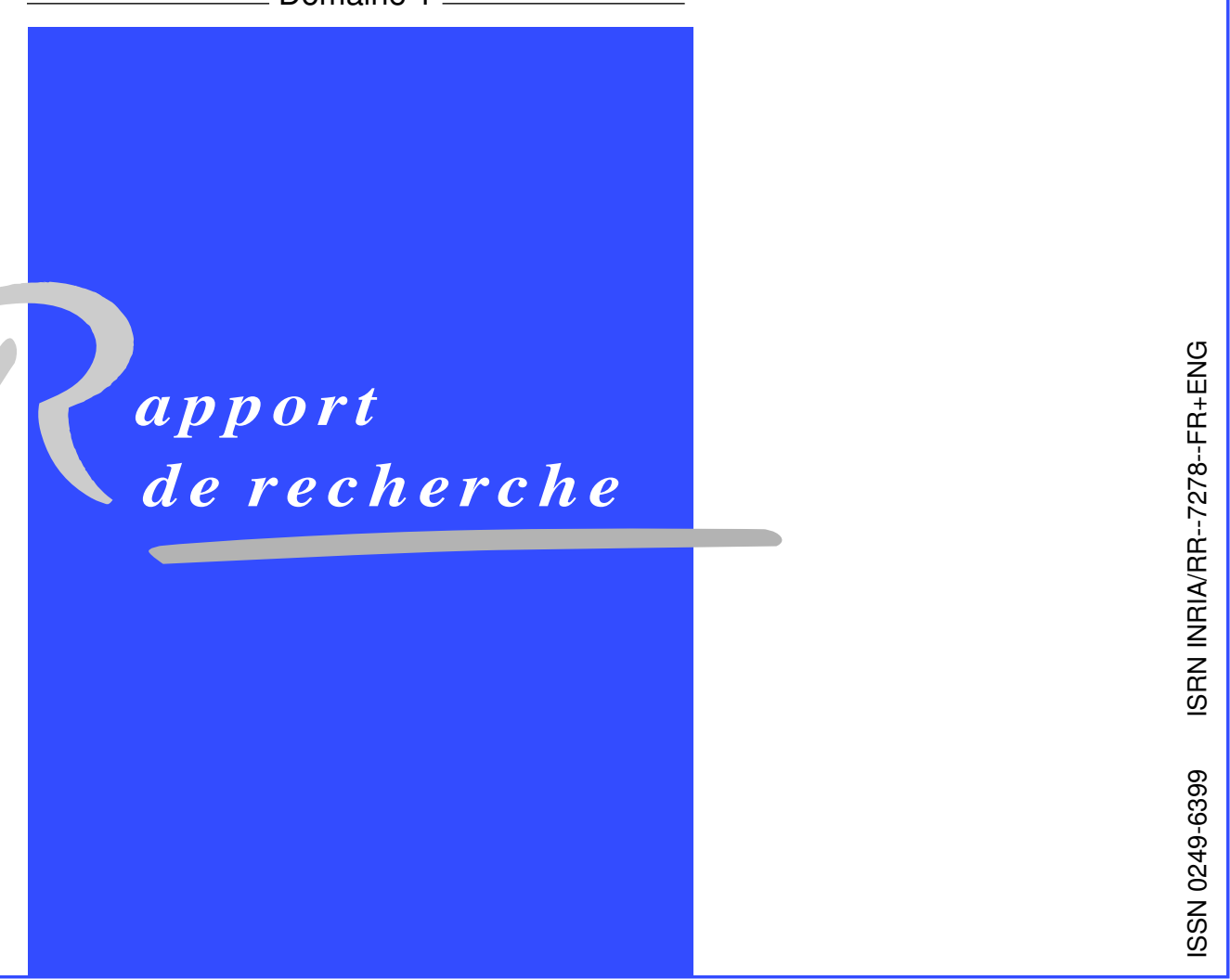





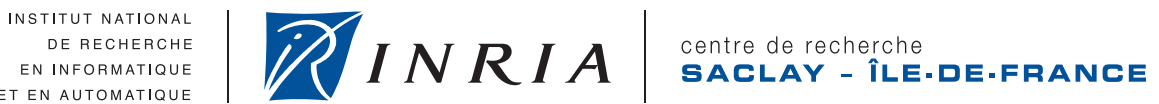

\title{
Exactly Solvable Stochastic Processes for Traffic Modelling
}

\author{
Maxim Samsonov* ${ }^{*}$, Cyril Furtlehner* , Jean-Marc Lasgouttes ${ }^{\dagger}$ \\ Domaine : Mathématiques appliquées, calcul et simulation \\ Équipes-Projets Imara et Tao \\ Rapport de recherche $\mathrm{n}^{\circ} 7278$ - Mai 2010 - 28 pages
}

\begin{abstract}
We investigate different available methods in the study of exactly solvable stochastic models and their application to the construction of models with acceleration/deceleration dynamics relevant to the road traffic modelling. We consider two family of models: one consisting in multi-speed exclusion processes with acceleration/braking transitions preserving pairwise neighbours locality; a second family of models consisting of tandem queues, with rates coupled dynamically to the flow of clients and enabling for a spontaneous hysteresis mechanism between acceleration and braking phases along the flow.
\end{abstract}

Key-words: Exclusion processes, queuing processes, integrable systems, fundamental diagram

\footnotetext{
* INRIA Saclay - LRI, Bat. 490, Université Paris-Sud - 91405 Orsay cedex (France)

$\dagger$ INRIA Paris Rocquencourt - Domaine de Voluceau B.P. 105 - 78153 Le Chesnay cedex (France)
}

Centre de recherche INRIA Saclay - Île-de-France

Parc Orsay Université

4, rue Jacques Monod, 91893 ORSAY Cedex

Téléphone : +33172925900 


\section{Processus Stochastiques exactement solubles et modélisation du Trafic}

Résumé : Nous passons en revue différentes méthodes dans l'étude des processus stochastiques exactement solubles, et leur application à la construction de modèles contenant une dynamique d'accélération/décélération cruciale dans la modélisation du traffic routier. Nous considérons deux familles de modèles: la première consiste en des processus d'exclusion à plusieurs types de particules avec des transitions d'accélérations/décélérations préservant la localité de voisinage imédiat de la dynamique stochastique; la seconde consiste en des systèmes de files d'attente assemblées en tandem, dont les taux de service sont couplés dynamiquement au flot des clients, permettant ainsi la mise en place spontannée d'un mechanisme d'hystéresis entre la phase d'accélération et de décélaration le long du flot.

Mots-clés : Processus d'exclusion, files d'attente, modèles intégrables, Diagramme Fondamental 


\section{Contents}

1 Introduction 3

2 Multi-speed exclusion processes 4

2.1 Model definition ................... . . . 4

2.2 Known special cases . . . . . . . . . . . . . . . . . 6

2.3 Relation to tandem queues ............... 6

2.4 Phenomenological observations . . . . . . . . . . . . 7

3 Exact solvability 9

3.1 Integrability . . . . . . . . . . . . . . . . . 9

3.2 Inter-particle generating functions . . . . . . . . . . . . . . . 11

3.3 Free fermions . . . . . . . . . . . . . . . . . . . 14

3.4 Matrix Ansatz . . . . . . . . . . . . . . . 15

4 Tandem queues with stochastic service rates 20

4.1 Model definition . . . . . . . . . . . . . . . . 20

4.2 Product form of clusters at steady-state . . . . . . . . . . . . 21

4.3 Examples for traffic models . . . . . . . . . . . . . . . . 22

4.4 Computing the fundamental diagram . . . . . . . . . . . . 25

5 Conclusion and Perspectives $\quad 27$

\section{Introduction}

In the study of models for traffic [1], properties of the fundamental diagram play an important role. The fundamental diagram of traffic flow gives a relation between the traffic flux (cars per unit of time) and the traffic density (cars per unit of length) or alternatively the dependence between the speed and the flux, or the speed and the density. These three quantities are related by the equation

$$
\text { flux }=\text { density } \times \text { speed. }
$$

In the three phases traffic theory of Kerner [2], the traffic diagram on highways consists of the free flow, the synchronized flow and the wide moving jam. In the free flow regime, at low density, the flux is simply proportional to the density of cars; in the congested one, at large density, massive clusters of cars are present, and the flux decreases more or less linearly with this density; in the intermediate regime, the relation between flux and density is largely of stochastic nature, due to the presence of a large amount of small clusters of cars propagating at various random speeds. It is not clear however whether in this picture these phases, and especially the synchronized flow phase, are genuine dynamical or thermodynamical phases, meaningful in some large size limit in the stationary regime, or are intricate transient features of a slowly relaxing system. In fact there is still controversy about the reality of the synchronized phase of Kerner at the moment [3].

Considering the large amount of successful applications of exactly solvable models to problems of non-equilibrium statistical physics [4], such a model applied to traffic could help to clarify this kind of questions. The idea is to apply 
the methods from the study of particle reactions with diffusion to problems of traffic, where such key facts can be observed, as clusterization (formation of condensates of cars), with the focus on the following questions:

- is there a stochastic model (exclusion process, zero range process) able to account for some or all reasonable features of the fundamental diagram observed experimentally, and suitable for exact computations? In particular, can we define a solvable model which takes into account the asymmetry between braking and accelerating, a property which plays a central role for the "good" behavior of the cellular automaton of Nagel-Schreckenberg [5] or its refined version like the velocity dependant randomized one [6] exhibiting a first order phase transition between the fluid and the congestion phase and hysteresis phenomena associated to metastable states.

- Having such a model, can we provide a method to compute the fundamental diagram and study emergence of non-trivial collective behaviors at macroscopic level, caused for example by some spontaneous symmetry breaking among identical vehicles that can be seen experimentally on a ring [7].

In addition, some mathematical properties of such models are worth investigating:

- the invariant measure can be non trivial, and the matrix ansatz solving the simple exclusion process [8] may provide the solution in some cases [9].

- in other cases, the invariant measure is simpler, for example because of some reversibility property, yielding e.g. product measure for queues, but also under some more general conditions which may be worth to explore.

- the integrability of such model is also an important question, because for such model the invariant measure and all excited states can be (in principle at least) constructed.

The paper is organized as follows: after defining the family of exclusion processes relevant to traffic, we discuss methods available in the study of the exactly solvable models, and formulate how an integrable version of the model should look like. We then analyze steady-state properties when a mapping with queuing process is possible.

\section{Multi-speed exclusion processes}

\section{$2.1 \quad$ Model definition}

The model we are interested in is a multi-type exclusion process, generalizing the simple exclusion process on the line, introduced by Spitzer in the 70's [10, 11], combined with some feature of the Nagel-Schreckenberg cellular automaton [5].

In the totally asymmetric version of the exclusion process (TASEP), particles move randomly on a 1-d lattice, always in the same direction, hopping from one site to the next within a time interval following a Poisson distribution and conditionally that the next site is vacant. In the Nagel-Schreckenberg cellular automaton, the dynamics is parallel: all particles update their positions at fixed 
time intervals, but their speeds are encoded in the number of steps that they can take. This speed can adapt stochastically, depending on the available space in front of the particle.

The model that we propose combines the braking and accelerating feature of the Nagel-Schreckenberg models, with the locality of the simple ASEP model, in which only two consecutive sites do interact at a given time. The trick is to allow each car to change stochastically its hopping rate, depending on the state of the next site. For a 2-speed model, let $A$ denotes sites occupied by fast vehicles, $B$ sites occupied by slow ones and let $O$ denote empty sites. Then the model we propose is defined by the following set of reactions, involving pairs of neighbouring sites:

$$
\begin{array}{ll}
A O \stackrel{\lambda_{a}}{\longrightarrow} O A & \text { simple move of fast vehicle } \\
B O \stackrel{\lambda_{b}}{\longrightarrow} O B & \text { simple move of slow vehicle } \\
B O \stackrel{\gamma_{b o}}{\longrightarrow} A O & \text { slow vehicle spontaneously accelerates } \\
A B \stackrel{\delta_{a b}}{\longrightarrow} B B & A \text { brakes behind } B \\
A A \stackrel{\delta_{a a}}{\longrightarrow} B A & A \text { brakes behind } A \\
A B \stackrel{\lambda_{a b}}{\longrightarrow} B A & A \text { overtakes } B
\end{array}
$$

Other possible transitions, less relevant from the point of view of traffic modelling might be useful when discussing integrability:

$$
\begin{array}{ll}
A O \stackrel{\delta_{a o}}{\longrightarrow} B O & \text { fast vehicle spontaneously decelerates } \\
B A \stackrel{\delta_{b a}}{\longrightarrow} B B & A \text { brakes in front of } B \\
A B \stackrel{\gamma_{a b}}{\longrightarrow} A A & B \text { accelerates in front of } A \\
B B \stackrel{\gamma_{b b}^{1}}{\longrightarrow} B A & B \text { accelerates in front of } B \\
B A \stackrel{\gamma_{b a}}{\longrightarrow} A A & B \text { accelerates behind } A \\
B B \stackrel{\gamma_{b b}^{2}}{\longrightarrow} A B & B \text { accelerates behind } B
\end{array}
$$

The $\lambda$ 's, $\gamma$ 's and $\delta$ 's denote the transition rates, each transition corresponding to a Poisson event. The dynamics is therefore purely sequential as opposed to the dynamics of the Nagel-Schreckenberg model. The first set of transitions correspond to normal movements of vehicles. They encode the fact that a slow vehicle tends to accelerate when there is space ahead, while in the opposite case, corresponding to (4) and (5) it tends slow down. Finally, (6) leaves fast cars the possibility to overtake slow cars. The main mechanism behind congestion, namely the asymmetry between braking and acceleration is therefore potentially present in the model when $\gamma_{b o}$ is different from $\delta_{a a}$ and $\delta_{a b}$.

To define fully the model, its boundary conditions have to be specified, either periodic in the ring geometry or with edges. In the latter case, additional 
incoming and outgoing rates have to be specified, depending on whether we want to model a traffic light a stop or simply a segment of highway for example.

The main question addressed in this paper is how to identify particular settings of the transition rates, with some of them possibly vanishing, such that the model becomes solvable in a broad sense, i.e. for which either the whole dynamics can be mathematically solved when the model is integrable, or at least that the stationary state can be described explicitly with the invariant measure.

\subsection{Known special cases}

Let us first notice that this model contains and generalizes several sub-models which are known to be integrable with particular rates. The diffusion part $(1,2)$ of the models is just the totally asymmetric exclusion process $[10,11]$ (TASEP) when $\lambda_{a}=\lambda_{b}$, which is known to be integrable. Its generalization to include multiparticle dynamics with overtaking is the so-called Karimipour model $[12,13]$ when $\lambda_{a b}=\lambda_{a}-\lambda_{b}$, which turns out to be integrable as well. Matrix Ansatz method allows in some cases, like the ASEP with open boundary conditions, to describe the stationary regime of the models, using the representations of the so-called diffusion algebras [8]. The acceleration/deceleration dynamics is equivalent to the coagulation/decoagulation models, which are known to be solvable by the empty interval method and by free fermions for particular sets of rates. Thus, a natural candidate for integrable model would be a combination of these two models, which we investigate further. To help investigating integrability, we developed a Java symbolic package for working with the stochastic integrable models.

\subsection{Relation to tandem queues}

In some cases, the model can be exactly reformulated in terms of generalized queueing processes (or zero range processes in the statistical physics parlance), where service rates of each queue follows as well a stochastic dynamics [14]. In this preceding work, however, we considered exclusion processes involving three consecutive sites interactions. In fact, in the present case, a large family of sub-models can be mapped onto such queueing processes. The mapping works only on the ring geometry, by identifying queues either with

(i) cars: clients are the empty sites.

(ii) empty sites: clients are the vehicles;

To get the mapping of type (i), it is simply needed that transitions involving a particle of type A or B is solely conditioned to whether the next site is empty (there is at least one client in the queue) or occupied (the queue is empty). This happens when

$$
\left\{\begin{array}{l}
\lambda_{a b}=0 \\
\delta_{a a}=\delta_{a b} \stackrel{\text { def }}{=} \delta \\
\gamma_{a b}=\gamma_{b b}^{1}=0 \\
\gamma_{b a}=\gamma_{b b}^{2} \stackrel{\text { def }}{=} \gamma
\end{array}\right.
$$


The resulting model then describe vehicles which accelerate when there is space ahead and decelerate when the next site is occupied. In the corresponding queuing process, queues are associated either to fast or slow cars, having then service rates $\lambda_{a}$ or $\lambda_{b}$. Slow queues become fast at rate $\delta$ conditionally to having at least one client, while empty fast queues become slow at rate $\gamma$.

The mapping of type (ii) is not possible with transitions limited to 2-consecutive sites interactions, because in that case homogeneity is not maintained in the clusters, and additional information to the number of cars and the rate of the car leaving the queue is needed to know the service rate of the queue. A way to circumvent this, is to start from a a slightly different definition of our initial model, by not attaching speed labels to cars, but instead to empty sites. Let $A$ and $B$ denote empty site fast and slow speed, and $V$ sites occupied with a vehicle, the set of transitions reads then

$$
\begin{array}{ll}
V A \stackrel{\lambda_{a}}{\longrightarrow} A V & \text { simple move of fast vehicle } \\
V B \stackrel{\lambda_{b}}{\longrightarrow} B V & \text { simple move of slow vehicle } \\
V A \stackrel{\delta}{\longrightarrow} V B & \text { fast site become } \\
\bar{V} B \stackrel{\gamma}{\longrightarrow} \bar{V} A & \text { slow site become fast }
\end{array}
$$

where $\bar{V}$ is an unoccupied site i.e. either $A$ or $B$.

The mechanism in this model is that the empty sites visited more recently have a slower associated speed than others, leading possibly to congestion instability. The mapping then to tandem queues is then straightforward: queues are associated to empty site of type $A$ or $B$ with corresponding service rate $\lambda_{a}$ or $\lambda_{b}$; fast queues with at least one client have a probability per unit of time $\delta$ to become a slow queue, while slow queue which are empty become fast with probability rate $\gamma$. This model generalizes directly to any speed levels. Interestingly, one see that in such a model that the service rate of queues are somehow related to the lifetime of clusters.

\subsection{Phenomenological observations}

Based on numerical simulations on the ring geometry, we make some observations concerning the phenomenology of a simplified form of the model, depending on the parameters. This is illustrated on Figure 1. Non-zero rates are $\lambda_{a}$ and $\lambda_{b}, \gamma_{b o}=\gamma, \delta_{a a}=\delta_{a b}=\delta$, while all others are set to zero. In particular, overtaking is excluded $\left(\lambda_{a b}=0\right)$.

When no asymmetry between braking and accelerating is present, as in TASEP on a ring, no spontaneous jam formation occurs. As the density $\rho$ of cars increases, one observes a smooth transition between a TASEP of fast particles for small $\rho$ to a TASEP of slow particles around $\rho \simeq 1$. Instead, when the ratio $\gamma / \delta$ is reduced, there is a proliferation of small jams. Below some threshold of this ratio, we observe a condensation phenomenon, associated to some critical value of the density: above this critical value, after a time which presumably scale like $L^{\alpha}$, one or more large jams, which absorb a finite fraction of the total number of cars, develop. If there are many of such large jams, a competition occurs, which end up in the long term with one single large condensate. This condensation mechanism is understood at equilibrium, combining 


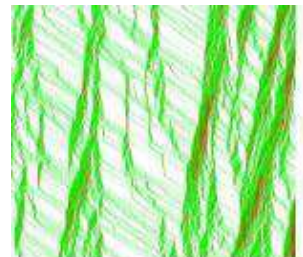

(a)

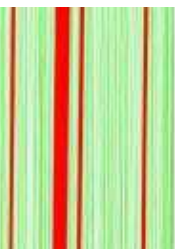

(b)

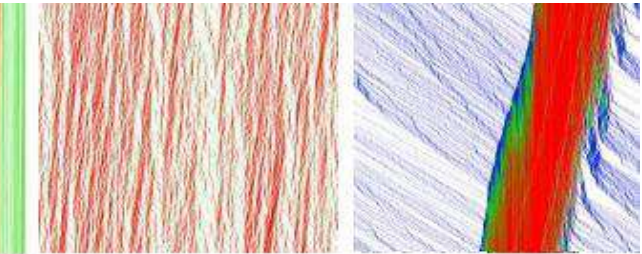

(c) (d)

Figure 1: Spate-time diagram for multi-speed exclusion process with 2 speed levels (a, b, c) and with 3 speed levels (d). Time is going downward and particles to the right. Red, green and blue represent different speeds in increasing order. The size of the system is 3000 except for (b) where it is 100000 . Setting are $\lambda_{a}=100, \lambda_{b}=10, \gamma_{a}=100, \delta_{b}=2$ for (a) and (b) and $\delta_{b}=10$ for (c), all with density $\rho=0.2$. In $(\mathrm{d})$ the rates are $\lambda_{c}=10, \lambda_{b}=100$ and $\lambda_{a}=200, \delta_{c}=3$, $\delta_{b}=5, \gamma_{b}=0.1$ and $\gamma_{a}=1$ with $\rho=0.3$.

the Nagel-Paczuski [15] interpretation of competing queues with some results in a previous work [14], which in the context of tandem queues on a ring, allows this condensation mechanism to occur if the apparition of slow vehicle is a sufficiently rare event.

In our context the mechanism for congestion is understood as follows: assume that a jam of size $s$ appears, created somewhere by a mutation $A \longrightarrow B$. Let $t(s)$ be the expected waiting time in this queue. The probability that a vehicle is still of type $A$ when it reaches the end of the queue is simply

$$
p\left(v=\lambda_{a}\right)=e^{-\delta t(s)}=p_{s} .
$$

Assuming that $s$ does not change much during $t(s)$, then we have

$$
t(s)=s\left(p_{s} / \lambda_{b}+\left(1-p_{s}\right) / \lambda_{a}\right),
$$

giving $t(s)$ self-consistently. This yields qualitatively that, at the beginning of the process, there is a distribution of jams with various effective service rates. As time evolves, either long-lived jams with effective service rates $1 / \lambda_{b}$ are able to survive, then new small jams can never appear because their effective service rates are strictly larger, so in the end there is a competition between the existing jams; the largest one finally remains alone after an exponentially large amount of time. If the situation with a single jam is not stable, which is observed below some critical density, then no large jam may develop at all, and only small fluctuations are to be observed. Typically this occurs when $\delta \ll \lambda_{b}$ and $\delta<\gamma$ (Figure $1(\mathrm{~b})$ ). In contrary when $>\delta>\gamma$ one likely observes the kind of jams in Figure 1(c).

We have also simulated a model with 3 speed levels, see Figure 1(d). In that case, small jams may have different speeds, depending on which type of slow car is leading. Then a cascade mechanism takes place, slow speed regions generate even slower speed clusters of cars and so on, and some kind of synchronized flow is observed.

To conclude this section, let us finally remark that Figure 1(a) is very reminiscent of coagulation-decoagulation process, which is somewhat expected from the previous discussion, since it is present in the equations. One could therefore 
expect the jam structure to share some properties with the directed percolation process taking place on the ring geometry.

\section{Exact solvability}

\subsection{Integrability}

In order to understand what exactly solvable models of statistical physics are good for, we consider the way calculations can be conducted with our proposed models and get some formulas of interest. The integrability means that we can construct the spectrum and the eigenstates of the corresponding Markov operator governing the evolution of the probability distribution $P(\mathcal{C}, t)$ with time:

$$
\frac{d}{d t} P(\mathcal{C}, t)=\sum_{\mathcal{C}^{\prime}} P\left(\mathcal{C}^{\prime}, t\right) M\left(\mathcal{C}^{\prime}, \mathcal{C}\right)-\sum_{\mathcal{C}^{\prime}} P(\mathcal{C}, t) M\left(\mathcal{C}, \mathcal{C}^{\prime}\right)
$$

where the element $M\left(\mathcal{C}^{\prime}, \mathcal{C}\right)$ of the Markov Matrix is the transitions rate between configuration $\mathcal{C}$ and $\mathcal{C}^{\prime}$. To the set of configuration, can be conveniently associated a tensor structure. Each particular state at a given site, like e.g. type A or B particle or empty site, is represented by an independent vector, and the tensor product run over the set of sites to represent a configuration. We then associate to the Markov Matrix an operator on this space:

$$
M=\sum_{\mathcal{C}, \mathcal{C}^{\prime}} M\left(\mathcal{C} \rightarrow \mathcal{C}^{\prime}\right)\left(\left(\text { transition operator for } \mathcal{C} \rightarrow \mathcal{C}^{\prime}\right)-\operatorname{Proj}(\mathcal{C})\right) .
$$

Let $L$ be a number of sites, with 3 possible states per sites, the model can be written in terms of pairs of creation/annihilation operators $\left(a_{i}, a_{i}^{\dagger}\right)$ and $\left(b_{i}, b_{i}^{\dagger}\right)$ on each site $i$ (with $\dagger$ corresponding here simply to transposition), given by the three-state matrices (acting only on $i$, reducing to the identity otherwise):

$$
a_{i}^{\dagger}=\left(\begin{array}{ccc}
0 & 0 & 1 \\
0 & 0 & 0 \\
0 & 0 & 0
\end{array}\right) \quad b_{i}^{\dagger}=\left(\begin{array}{ccc}
0 & 0 & 0 \\
0 & 0 & 1 \\
0 & 0 & 0
\end{array}\right) .
$$

with $n_{i}^{a} \stackrel{\text { def }}{=} a_{i}^{\dagger} a_{i}$ [resp. $n_{i}^{b} \stackrel{\text { def }}{=} b_{i}^{\dagger} b_{i}$ ] being the operator counting the number of particles (0 or 1$)$ of type $A$ [resp. of type $B$ ] on site $i$. Equipped with such notations, we rewrite the Markov operator in three different parts.

Hopping/Overtaking part of model dynamics:

$$
\begin{gathered}
A O \stackrel{\lambda_{a}}{\longrightarrow} O A \\
B O \stackrel{\lambda_{b}}{\longrightarrow} O B \\
A B \stackrel{\lambda_{a b}}{\longrightarrow} B A \\
M^{h o p}=\lambda_{a} \sum_{i}\left(a_{i} a_{i+1}^{+}-n_{i}^{a}\left(1-n_{i+1}^{a}-n_{i+1}^{b}\right)\right) \\
+\lambda_{b} \sum_{i}\left(b_{i} b_{i+1}^{+}-n_{i}^{b}\left(1-n_{i+1}^{a}-n_{i+1}^{b}\right)\right)+\lambda_{a b} \sum_{i}\left(b_{i}^{+} a_{i} a_{i+1}^{+} b_{i+1}-n_{i}^{a} n_{i+1}^{b}\right)
\end{gathered}
$$




\section{Coagulation part of model dynamics:}

$$
\begin{aligned}
& A A \stackrel{\delta_{a a}^{1}}{\longrightarrow} B A \quad A \text { breaks behind } A \\
& A A \stackrel{\delta_{a a}^{2}}{\longrightarrow} A B \quad A \text { breaks before } A \\
& B B \stackrel{\gamma_{b b}^{1}}{\longrightarrow} B A \quad B \text { accelerates before } B \\
& B B \stackrel{\gamma_{b b}^{2}}{\longrightarrow} A B \quad B \text { accelerates behind } B \\
& M^{\text {coag }}=\delta_{a a}^{1} \sum_{i}\left(b_{i}^{+} a_{i} n_{i+1}^{a}-n_{i}^{a} n_{i+1}^{a}\right)+\delta_{a a}^{2} \sum_{i}\left(n_{i}^{a} b_{i+1}^{+} a_{i+1}-n_{i}^{a} n_{i+1}^{a}\right)+ \\
& +\gamma_{b b}^{1} \sum_{i}\left(n_{i}^{b} a_{i+1}^{+} b_{i+1}-n_{i}^{b} n_{i+1}^{b}\right)+\gamma_{b b}^{2} \sum_{i}\left(a_{i}^{+} b_{i} n_{i+1}^{b}-n_{i}^{b} n_{i+1}^{b}\right)
\end{aligned}
$$

\section{Decoagulation part of model dynamics:}

$$
\begin{gathered}
A B \stackrel{\delta_{a b}}{\longrightarrow} B B \quad A \text { breaks behind } B \\
B A \stackrel{\delta_{b a}}{\longrightarrow} B B \quad A \text { breaks before } B \\
A B \stackrel{\gamma_{a b}}{\longrightarrow} A A \quad B \text { accelerates before } A \\
B A \stackrel{\gamma_{b a}}{\longrightarrow} A A \quad B \text { accelerates behind } A \\
M^{\text {decoag }}=\quad \begin{array}{l}
\delta_{a b} \sum_{i}\left(b_{i}^{+} a_{i} n_{i+1}^{b}-n_{i}^{a} n_{i+1}^{b}\right)+\delta_{b a} \sum_{i}\left(n_{i}^{b} b_{i+1}^{+} a_{i+1}-n_{i}^{b} n_{i+1}^{a}\right)+ \\
+\gamma_{a b} \sum_{i}\left(n_{i}^{a} a_{i+1}^{+} b_{i+1}-n_{i}^{a} n_{i+1}^{b}\right)+\gamma_{b a} \sum_{i}\left(a_{i}^{+} b_{i} n_{i+1}^{a}-n_{i}^{b} n_{i+1}^{a}\right)
\end{array}
\end{gathered}
$$

There is a special choice of the rates such that the terms $n_{i}^{x} n_{i+1}^{y}$ disappears, provided that we have the following relation between the rates:

$$
\lambda_{a}=\delta_{a a}^{1}+\delta_{a a}^{2}, \quad \lambda_{b}=\gamma_{b b}^{1}+\gamma_{b b}^{2}=\delta_{b a}+\gamma_{b a}, \quad \lambda_{a b}=\delta_{a a}^{1}+\delta_{a a}^{2}-\delta_{a b}-\gamma_{a b}
$$

We rewrite then the Hamiltonian in the following convenient form:

$$
H=-\sum_{i=1}^{L} e_{i}
$$

with

$$
\begin{aligned}
e_{i} & =\lambda_{a} a_{i} a_{i+1}^{+}+\lambda_{b} b_{i} b_{i+1}^{+}+\lambda_{a b} b_{i}^{+} a_{i} a_{i+1}^{+} b_{i+1} \\
& -\lambda_{a} n_{i}^{a}-\lambda_{b} n_{i}^{b}+n_{i}^{a}\left(\delta_{a a}^{2} b_{i+1}^{+} a_{i+1}+\gamma_{a b} a_{i+1}^{+} b_{i+1}\right)+n_{i}^{b}\left(\delta_{b a} b_{i+1}^{+} a_{i+1}+\gamma_{b b}^{1} a_{i+1}^{+} b_{i+1}\right) \\
& +\left(\delta_{a a}^{1} b_{i}^{+} a_{i}+\gamma_{b a} a_{i}^{+} b_{i}\right) n_{i+1}^{a}+\left(\delta_{a b} b_{i}^{+} a_{i}+\gamma_{b b}^{2} a_{i}^{+} b_{i}\right) n_{i+1}^{b} .
\end{aligned}
$$

The next step then is to see at which condition the operators $e_{i}$ are elements of an Hecke algebra, namely verifying up to a scalar shift the following TempereleyLieb relationship:

$$
e_{i} e_{i+1} e_{i}=e_{i+1} e_{i} e_{i+1} \text {. }
$$

Let us check this first for a simpler case, the Karimipour-Model and then try to improve the obtained relations by including the appropriate reactions. For the aforementioned case we have the following elements:

$$
f_{i}=\left(f_{1}\right)_{i}-\left(f_{2}\right)_{i}=\lambda_{a} a_{i} a_{i+1}^{+}+\lambda_{b} b_{i} b_{i+1}^{+}+\lambda_{a b} b_{i}^{+} a_{i} a_{i+1}^{+} b_{i+1}-\lambda_{a b} n_{i}^{a} n_{i+1}^{b}
$$


Then we have the following relations:

$$
\begin{aligned}
\left(f_{1}\right)_{i}\left(f_{1}\right)_{i+1}=\lambda_{a}^{2} a_{i} n_{i+1}^{a} a_{i+2}^{+} & +\lambda_{b}^{2} b_{i} n_{i+1}^{b} b_{i+2}^{+}+\lambda_{a b}^{2} b_{i}^{+} a_{i} n_{i+1}^{a} a_{i+2}^{+} b_{i+2} \\
& +\lambda_{a} \lambda_{b}\left(a_{i} a_{i+1}^{+} b_{i+1} b_{i+2}+b_{i} b_{i+1}^{+} a_{i+1} a_{i+2}\right) \\
\left(f_{1}\right)_{i+1}\left(f_{1}\right)_{i}=\lambda_{a}^{2} a_{i} n_{i+1}^{e} a_{i+2}^{+} & +\lambda_{b}^{2} b_{i} n_{i+1}^{e} b_{i+2}^{+}+\lambda_{a b}^{2} b_{i}^{+} a_{i} n_{i+1}^{b} a_{i+2}^{+} b_{i+2} \\
& +\lambda_{a} \lambda_{a b}\left(b_{i}^{+} a_{i} b_{i+1} a_{i+2}+a_{i} b_{i+1}^{+} a_{i+2}^{+} b_{i+2}\right)
\end{aligned}
$$

and as well we have the following relation:

$$
\left(f_{1}\right)_{i+1}\left(f_{1}\right)_{i}\left(f_{1}\right)_{i+1}=0
$$

To include input of particles at the end points we add the boundary terms into the Markov Matrix:

$-H^{1}-H^{L}=\alpha\left(a_{1}^{+}-n_{1}^{a}\right)+\beta\left(b_{1}^{+}-n_{1}^{b}\right)+\alpha^{\prime}\left(a_{L}-1+n_{L}^{a}+n_{L}^{b}\right)+\beta^{\prime}\left(b_{L}-1+n_{L}^{a}+n_{L}^{b}\right)$

For the TASEP we have the following $R$-matrices which guarantee the integrability and as well the Tempereley-Lieb relations between the generators $e_{i}$ :

$$
R(u, v)=\frac{u}{u-v} \mathcal{P}+\left(\begin{array}{cccc}
0 & 0 & 0 & 0 \\
0 & 1 & 0 & 0 \\
0 & -1 & 0 & 0 \\
0 & 0 & 0 & 0
\end{array}\right)
$$

and as well the following:

$$
R(u, v)=\frac{1-p u-q v+p q u v}{u-v} \mathcal{P}+\left(\begin{array}{cccc}
0 & 0 & 0 & 0 \\
0 & p-q & 0 & 0 \\
0 & -p+q & 0 & 0 \\
0 & 0 & 0 & 0
\end{array}\right)
$$

\subsection{Inter-particle generating functions}

The empty interval method and its generalizations was successfully applied to solve multiparticle dynamics problems. The method allowed to bypass the YangBaxter equation, by adjusting the rates of reactions. The method as well was further extended to be applied to the systems on graphs. In view of this, we will combine the Bethe-Ansatz with the generalization of the empty interval method to get a more complex set of models solvable by this approach. We will consider the generating functions of the form:

$\mathcal{F}[\Psi(t), x, x+m-1]=\left\langle\Omega\left|\prod_{i=x}^{x+m-1}\left(A(i) n_{i}^{a}+B(i) n_{i}^{b}+E(i)\left(1-n_{i}^{a}-n_{i}^{b}\right)\right)\right| \Psi(t)\right\rangle$,

where

$$
\frac{d}{d t} \Psi(t)=-H \Psi(t)
$$

and $\langle\Omega| H=0$. In case of the TASEP we have the following Hamiltonian:

$$
H=\sum_{i}\left(\sigma_{i+1}^{+} \sigma_{i}^{-}-n_{i}\left(1-n_{i+1}\right)\right)
$$

RR $n^{\circ} 7278$ 
The introduced functions can be rewritten as well as

$$
\left\langle\Omega\left|\prod_{i=x}^{x+m-1}\left(C(i)+D(i) \sigma_{i}^{z}\right)\right| \Psi(t)\right\rangle
$$

For the function $\mathcal{F}[\Psi(t), x, x+m-1]$ we get the relation equation:

$$
\frac{d}{d t} \mathcal{F}[\Psi(t), x, x+m-1]=\left\langle\Omega\left|\left[H, \prod_{i=x}^{x+m-1}\left(C(i)+D(i) \sigma_{i}^{z}\right)\right]\right| \Psi(t)\right\rangle
$$

Then this equation has the following form:

$$
\begin{aligned}
& \frac{d}{d t} \mathcal{F}[\Psi(t), x, x+m-1]= \\
& \sum_{k=x+1}^{x+m-2}(A(k)-B(k))\left\langle\Omega\left|\left(\sigma_{k+1}^{+} \sigma_{k}^{-}-\sigma_{k}^{+} \sigma_{k-1}^{-}\right) \prod_{i=x, i \neq k}^{x+m-1}\left(A(i) n_{i}+E(i)\left(1-n_{i}\right)\right)\right| \Psi(t)\right\rangle \\
& +(A(x)-B(x))\left\langle\Omega\left|\sigma_{x+1}^{+} \sigma_{x}^{-} \prod_{i=x+1}^{x+m-1}\left(A(i) n_{i}+E(i)\left(1-n_{i}\right)\right)\right| \Psi(t)\right\rangle \\
& -(A(x+m-1)-B(x+m-1))\left\langle\Omega\left|\sigma_{x+m-1}^{+} \sigma_{x+m-2}^{-} \prod_{i=x}^{x+m-2}\left(A(i) n_{i}+E(i)\left(1-n_{i}\right)\right)\right| \Psi(t)\right\rangle
\end{aligned}
$$

We will consider as well another type of the functions, which we call the amplitudes of fermionic holes in the vacuum $\Omega$. By definition the fermionic one hole amplitude is defined as the following:

$$
\mathcal{F}_{k}[\Psi(t)]=\left\langle\Omega\left|\prod_{i=1}^{k-1} \sigma_{i}^{z} \sigma_{k}^{+}\right| \Psi(t)\right\rangle
$$

Let us consider first only the coagulation/decoagulation part of the model. This model can be dealt with using the empty interval method [16]. Let us imagine a densely packed road with cars of two types with open boundary conditions. Then we can write a system of equations for the queues of cars in terms of usual probability measures

$$
\begin{aligned}
P_{t}^{a, b}(x, x+1, \ldots, x+m-1) & =\mathbb{E}^{t}\left(n_{x}^{a, b} n_{x+1}^{a, b} \cdots n_{x+m-1}^{a, b}\right) \\
& =\sum_{X, Y} \mathbb{E}^{t}\left(X n_{x}^{a, b} n_{x+1}^{a, b} \cdots n_{x+m-1}^{a, b} Y\right)
\end{aligned}
$$

where $X$ and $Y$ are all monomials created from $n_{y}^{a}$ and $n_{z}^{b}$ such that length $(X)+$ length $(Y)=N-m$. Monte-Carlo modelling consists in choosing pairs of neighboring cars and then updating them according to the proposed dynamical rules with the probability (rate) $\Delta t /(N-1)$ for open boundary conditions. Taking into account the Markovian evolution, the equations of the corresponding pro- 
cess can be written in the following form:

$$
\begin{aligned}
& \frac{d}{d t} P_{t}^{a}(x, \ldots, x+m-1)= \\
& \quad \lambda_{a b}\left(\mathbb{E}^{t}\left(n_{x-1}^{a} n_{x}^{b} n_{x+1}^{a} \ldots n_{x+m-1}^{a}\right)-\mathbb{E}^{t}\left(n_{x}^{a} n_{x+1}^{a} \ldots n_{x+m-1}^{a} n_{x+m}^{b}\right)\right)+ \\
& \quad+\left(\gamma_{a b}+\gamma_{b a}\right) \sum_{y=x}^{x+m-3} \mathbb{E}^{t}\left(n_{x}^{a} \cdots n_{y}^{a} n_{y+1}^{b} n_{y+2}^{a} \ldots n_{x+m-1}^{a}\right)+ \\
& +\gamma_{a b} \mathbb{E}^{t}\left(n_{x}^{a} \cdots n_{x+m-2}^{a} n_{x+m-1}^{b}\right)+\gamma_{a b} \mathbb{E}^{t}\left(n_{x-1}^{a} n_{x}^{b} n_{x+1}^{a} \cdots n_{x+m-1}^{a}\right)+ \\
& +\gamma_{b a} \mathbb{E}^{t}\left(n_{x}^{b} n_{x+1}^{a} \cdots n_{x+m-1}^{a}\right)+\gamma_{b a} \mathbb{E}^{t}\left(n_{x}^{a} n_{x+1}^{a} \cdots n_{x+m-1}^{b} n_{x+m}^{a}\right)+ \\
& +\gamma_{b b}^{1} \mathbb{E}^{t}\left(n_{x-1}^{b} n_{x}^{b} n_{x+1}^{a} \cdots n_{x+m-1}^{a}\right)+\gamma_{b b}^{2} \mathbb{E}^{t}\left(n_{x}^{a} \cdots n_{x+m-1}^{b} n_{x+m}^{b}\right)- \\
& -(m-1)\left(\delta_{a a}^{1}+\delta_{a a}^{2}\right) \mathbb{E}^{t}\left(n_{x}^{a} n_{x+1}^{a} \cdots n_{x+m-1}^{a}\right)-\delta_{a a}^{1} \mathbb{E}^{t}\left(n_{x}^{a} n_{x+1}^{a} \cdots n_{x+m}^{a}\right)- \\
& -\delta_{a a}^{2} \mathbb{E}^{t}\left(n_{x-1}^{a} n_{x}^{a} n_{x+1}^{a} \cdots n_{x+m}^{a}\right)-\delta_{a b} \mathbb{E}^{t}\left(n_{x}^{a} \cdots n_{x+m-1}^{a} n_{x+m}^{b}\right)- \\
& -\delta_{b a} \mathbb{E}^{t}\left(n_{x-1}^{b} n_{x}^{a} \cdots n_{x+m-1}^{a}\right) .
\end{aligned}
$$

Rewriting these equations in terms of queues of cars of type $a$, using $n_{x}^{a}+n_{x}^{b}=$ 1 , we get the following equations:

$$
\begin{aligned}
& \frac{d}{d t} P_{t}^{a}(x, \ldots, x+m-1)= \\
& \quad\left(\delta_{b a}+\gamma_{b b}^{1}-\lambda_{a b}-\gamma_{a b}\right) P_{t}^{a}(x-1, \ldots, x+m-1)+ \\
& \quad+\left(\lambda_{a b}+\gamma_{a b}-\gamma_{b b}^{1}\right) P_{t}^{a}(x-1, x+1, \ldots, x+m-1)- \\
& \quad-\left(\lambda_{a b}+(m-1)\left(\delta_{a a}^{1}+\delta_{a a}^{2}+\gamma_{a b}+\gamma_{b a}\right)+\delta_{a b}+\delta_{b a}+\gamma_{b b}^{1}+\gamma_{b b}^{2}\right) \\
& \quad \times P_{t}^{a}(x, \ldots, x+m-1)+ \\
& \quad+\left(\lambda_{a b}-\gamma_{b a}+\gamma_{b b}^{2}-\delta_{a a}^{1}+\delta_{a b}\right) P_{t}^{a}(x, \ldots, x+m)+ \\
& \quad+\left(\gamma_{a b}+\gamma_{b a}\right) \sum_{y=x}^{x+m-3} P_{t}^{a}(x, \ldots, y, y+2, \ldots, x+m-1)+ \\
& \quad+\left(\gamma_{a b}+\gamma_{b b}^{2}\right) P_{t}^{a}(x, \ldots, x+m-2)+\left(\gamma_{b a}+\gamma_{b b}^{1}\right) P_{t}^{a}(x+1, \ldots, x+m-1)+ \\
& +\left(\gamma_{b a}-\gamma_{b b}^{2}\right) P_{t}^{a}(x, \ldots, x+m-2, x+m) .
\end{aligned}
$$

These equations can be solved for some particular choice of the rates just in terms of the functions of queues. When $\gamma_{a b}=\gamma_{b a}=\gamma_{b b}^{2}=0$ and $\lambda_{a b}=\gamma_{b b}^{1}$, we 
get the following equations:

$$
\begin{aligned}
\frac{d}{d t} P_{t}^{a}(x, y) & =\delta_{b a} P_{t}^{a}(x-1, y)-\left(2 \lambda_{a b}+(y-x)\left(\delta_{a a}^{1}+\delta_{a a}^{2}\right)+\delta_{a b}+\delta_{b a}\right) P_{t}^{a}(x, y)+ \\
& +\left(\lambda_{a b}-\delta_{a a}^{1}+\delta_{a b}\right) P_{t}^{a}(x, y+1)+\lambda_{a b} P_{t}^{a}(x+1, y) .
\end{aligned}
$$

Such equations are solved in terms of Bessel functions. In general one can consider the functions of the form

$$
Q_{t}^{a, b}(x, y)=\sum_{x \leq j_{1}<j_{2}<\ldots j_{p} \leq y} c_{j_{1}, j_{2}, \ldots, j_{p}}^{a, b} \mathbb{E}^{t}\left(n_{j_{1}}^{a, b} n_{j_{2}}^{a, b} \cdots n_{j_{p}}^{a, b}\right)
$$

and get a system of equations for more general values of rates. It turns out that more general stochastic processes can be solved using such functions, using the generalized inter-particle distribution function method.

\subsection{Free fermions}

Among the cases solvable by the method there are those solvable by the BetheAnsatz method and as well by the free-fermions, the anti-commuting field obtained via Jordan-Wigner transform. Let us consider the case solvable by the free fermions:

$$
\begin{array}{ll}
A B \stackrel{\lambda_{a b}}{\longrightarrow} B A & A \text { overtakes } B \\
B A \stackrel{\lambda_{b a}}{\longrightarrow} A B & B \text { overtakes } A \\
B B \stackrel{\lambda_{a b}}{\longrightarrow} B A & B \text { accelerates before } B \\
B B \stackrel{\lambda_{b a}}{\longrightarrow} A B & B \text { accelerates behind } B
\end{array}
$$

The corresponding free fermionic Hamiltonian looks as following:

$$
\begin{aligned}
H^{\text {freef }} & =\lambda_{a b} \sum_{i}\left(b_{i}^{+}\left(a_{i}+b_{i}\right) a_{i+1}^{+} b_{i+1}-2 n_{i}^{b} n_{i+1}^{a}\right) \\
& +\lambda_{b a} \sum_{i}\left(a_{i}^{+} b_{i} b_{i+1}^{+}\left(a_{i+1}+b_{i+1}\right)-2 n_{i}^{a} n_{i+1}^{b}\right)
\end{aligned}
$$

and the corresponding model of traffic would be: $H^{\text {freef }}+H^{\text {hop }}$. The strategy then is to analyze this model by considering $H^{\text {hop }}$ as a small perturbation. To include input of particles at the end points we add the boundary terms into the Markov Matrix:

$-H^{1}-H^{L}=\alpha\left(a_{1}^{+}-n_{1}^{a}\right)+\beta\left(b_{1}^{+}-n_{1}^{b}\right)+\alpha^{\prime}\left(a_{L}-1+n_{L}^{a}+n_{L}^{b}\right)+\beta^{\prime}\left(b_{L}-1+n_{L}^{a}+n_{L}^{b}\right)$

The question of integrability is how to construct an appropriate $R$-matrix for this kind of model? For the TASEP we have the following $R$-matrices:

$\mathrm{RR} \mathrm{n}^{\circ} 7278$

$$
R(u, v)=\frac{u}{u-v} \mathcal{P}+\left(\begin{array}{cccc}
0 & 0 & 0 & 0 \\
0 & 1 & 0 & 0 \\
0 & -1 & 0 & 0 \\
0 & 0 & 0 & 0
\end{array}\right)
$$


and as well the following:

$$
R(u, v)=\frac{1-p u-q v+p q u v}{u-v} \mathcal{P}+\left(\begin{array}{cccc}
0 & 0 & 0 & 0 \\
0 & p-q & 0 & 0 \\
0 & -p+q & 0 & 0 \\
0 & 0 & 0 & 0
\end{array}\right)
$$

\subsection{Matrix Ansatz}

The Matrix Product Ansatz is generated by the relations:

$$
H_{i, i+1}\left(\begin{array}{c}
A \\
B \\
E
\end{array}\right) \otimes\left(\begin{array}{c}
A \\
B \\
E
\end{array}\right)=X \otimes\left(\begin{array}{c}
A \\
B \\
E
\end{array}\right)-\left(\begin{array}{c}
A \\
B \\
E
\end{array}\right) \otimes X
$$

As well we need to fix the boundary vectors $\langle W|$ and $|V\rangle$ and the values of the operators $X_{1}, X_{2}, X_{3}$ on them:

$$
\left\langle W\left|\left(\begin{array}{c}
X_{1} \\
X_{2} \\
X_{3}
\end{array}\right)=\left\langle W\left|\left(\begin{array}{c}
x_{1} \\
x_{2} \\
x_{3}
\end{array}\right), \quad\left(\begin{array}{c}
X_{1} \\
X_{2} \\
X_{3}
\end{array}\right)\right| V\right\rangle=\left(\begin{array}{c}
x_{1}^{\prime} \\
x_{2}^{\prime} \\
x_{3}^{\prime}
\end{array}\right)\right| V\right\rangle
$$

In general, the operators $X_{i}$ are not supposed to act as scalars and depend on representation we chose. Due to cancellation of the bulk terms we get the following relations:

$$
\left\langle W\left|X=-\left\langle W\left|H^{1}\left(\begin{array}{c}
A \\
B \\
E
\end{array}\right), \quad X\right| V\right\rangle=H^{L}\left(\begin{array}{c}
A \\
B \\
E
\end{array}\right)\right| V\right\rangle
$$

explicitly we have:

$$
\left\langle W\left|X=\left\langle W\left|\left(\begin{array}{c}
\alpha \\
\beta \\
-\alpha-\beta
\end{array}\right), \quad X\right| V\right\rangle=\left(\begin{array}{c}
-\alpha^{\prime} A \\
-\beta^{\prime} B \\
\alpha^{\prime} A+\beta^{\prime} B
\end{array}\right)\right| V\right\rangle
$$

We get the following set of relations:

$$
\begin{aligned}
\left(\delta_{a a}^{1}+\delta_{a a}^{2}\right) A^{2}-\gamma_{b a} B A-\gamma_{a b} A B & =X_{1} A-A X_{1} \\
-\delta_{a a}^{1} A^{2}+\left(\delta_{b a}+\gamma_{b a}\right) B A-\lambda_{a b} A B-\gamma_{b b}^{1} B^{2} & =X_{2} A-B X_{1} \\
-\lambda_{a} A E & =X_{3} A-E X_{1} \\
-\delta_{a a}^{2} A^{2}+\left(\gamma_{a b}+\delta_{a b}+\lambda_{a b}\right) A B-\gamma_{b b}^{2} B^{2} & =X_{1} B-A X_{2} \\
-\delta_{b a} B A-\delta_{a b} A B+\left(\gamma_{b b}^{1}+\gamma_{b b}^{2}\right) B^{2} & =X_{2} B-B X_{2} \\
\lambda_{a} A E & =X_{1} E-A X_{3} \\
\lambda_{b} B E & =X_{2} E-B X_{3}
\end{aligned}
$$

First we consider the problem of construction the representations of the coagulation/decoagulation part, and then we extend it with diffusion. Thus we have the algebra:

$$
\begin{aligned}
\left(\delta_{a a}^{1}+\delta_{a a}^{2}\right) A^{2}-\gamma_{b a} B A-\gamma_{a b} A B & =X_{1} A-A X_{1} \\
-\delta_{a a}^{1} A^{2}+\left(\delta_{b a}+\gamma_{b a}\right) B A-\lambda_{a b} A B-\gamma_{b b}^{1} B^{2} & =X_{2} A-B X_{1} \\
-\delta_{a a}^{2} A^{2}+\left(\gamma_{a b}+\delta_{a b}+\lambda_{a b}\right) A B-\gamma_{b b}^{2} B^{2} & =X_{1} B-A X_{2} \\
-\delta_{b a} B A-\delta_{a b} A B+\left(\gamma_{b b}^{1}+\gamma_{b b}^{2}\right) B^{2} & =X_{2} B-B X_{2} \\
\text { RR n } \mathrm{n}^{\circ} 7278 \quad &
\end{aligned}
$$


A priori we have three types of representations: The scalar ones, the finite dimensional and the infinite dimensional. Let us first investigate the scalar representations, the elements $A$ and $B$ act as numbers and we have different compatibility conditions between the rates (to be presented shortly). For finite dimensional representations we have to look for representations of this algebra by assuming that $A+B$ is a diagonal $4 \times 4$ matrix and $A$ is upper triangular [17] for open boundary conditions. The dimension depends on the specific choice of the rates. In particular, for coagulation/decoagulation model with open right site and the incoming and outgoing particle rates at the first site, there has been constructed two-dimensional representation as well. And it turns out that in the finite dimensional case the elements of $X$ act as non-diagonal matrices. The matrix ansatz was constructed for the totally asymmetric coagulation model with the Hamiltonian $H=\sum_{j} h_{j, j+1}$ :

$$
h=\left(\begin{array}{cccc}
0 & 0 & 0 & 0 \\
0 & \Delta+1 & 0 & 0 \\
0 & -1 & 0 & -1 \\
0 & -\Delta & 0 & 1
\end{array}\right)
$$

This model is solvable by the free fermions and corresponds to the following asymmetric coagulation/decoagulation model:

$$
\begin{aligned}
& B B \stackrel{1}{\longrightarrow} B A \\
& A B \stackrel{\Delta}{\longrightarrow} B B \\
& A B \stackrel{1}{\longrightarrow} B A
\end{aligned}
$$

Consider as well the model

$$
\begin{aligned}
& B B \stackrel{\nu}{\longrightarrow} B A \\
& B B \stackrel{\mu}{\longrightarrow} A B \\
& A B \stackrel{1}{\longrightarrow} B A
\end{aligned}
$$

with Hamiltonian:

$$
\tilde{h}=\left(\begin{array}{cccc}
0 & 0 & 0 & 0 \\
0 & 1 & 0 & -\mu \\
0 & -1 & 0 & -\nu \\
0 & 0 & 0 & \mu+\nu
\end{array}\right)
$$


The corresponding $R$-matrix can be taken in the form:

$$
R(u, v)=\left(\begin{array}{cccc}
1 & 0 & 0 & 0 \\
0 & -a(u, v) & 1 & -c(u, v) \\
0 & a(u, v)+1 & 0 & -b(u, v) \\
0 & 0 & 0 & b(u, v)+c(u, v)+1
\end{array}\right)
$$

From the Yang-Baxter equation and the positivity of the rates it follows

$$
b(u, v)=0 \quad a(u, v)=\frac{u}{v}-1 .
$$

Thus the model is solvable for $\nu=1$ and $\mu=0$. The later model can be transformed with the matrix:

$$
U=\left(\begin{array}{cc}
1 & \frac{1}{2} \\
0 & \frac{1}{2}
\end{array}\right) \otimes\left(\begin{array}{cc}
1 & \frac{1}{2} \\
0 & \frac{1}{2}
\end{array}\right)
$$

to a model with annihilation:

$$
R(u, v)=\mathcal{P}+\left(\frac{u}{v}-1\right)\left(\begin{array}{cccc}
0 & 0 & 0 & -1 \\
0 & -1 & 0 & 0 \\
0 & 1 & 0 & 0 \\
0 & 0 & 0 & 1
\end{array}\right)
$$

Let us consider as well the model with the following $R$-matrix:

$$
R(u, v)=\left(\begin{array}{cccc}
1 & 0 & 0 & 0 \\
0 & -a(u, v) & 1 & -c(u, v) \\
0 & a(u, v)+b(u, v)+1 & 0 & 0 \\
0 & -b(u, v) & 0 & c(u, v)+1
\end{array}\right)
$$

Assume $c \neq 0$, then $a=c$ and $a=-b=\frac{u}{v}-1$. This case contradicts the positivity of the rates. Thus the limiting case of the model is not integrable, but the model is still solvable by the empty interval method. For the three type of particle we will consider the following model, which we conjecture to be integrable as well, for certain choice of the rates:

$$
\begin{aligned}
& A O \stackrel{\lambda_{a}}{\longrightarrow} O A \\
& B O \stackrel{\lambda_{b}}{\longrightarrow} O B \\
& A B \stackrel{\lambda_{a b}}{\longrightarrow} B A \\
& B B \stackrel{\delta}{\longrightarrow} A A
\end{aligned}
$$

This model generalizes the model of L. Cantini, to include the acceleration dynamics, as well we will investigate the model with deceleration when:

$$
A A \stackrel{\delta}{\longrightarrow} B B
$$


This is particularly interesting as we will investigate the integrability of these models as they map to a coagulation/decoagulation models

$$
\begin{aligned}
& A X_{1}=X_{1} A \\
& (\Delta+1) A B=X_{1} B-A X_{2} \\
& B^{2}+A B=B X_{1}-X_{2} A \\
& B^{2}-\Delta A B=X_{2} B-B X_{2}
\end{aligned}
$$

Let us describe the representation of this algebra, if we assume that $X_{i}$ act as numbers then we have:

$$
\begin{aligned}
& (\Delta+1) A B=x_{1} B-x_{2} A \\
& B^{2}=\Delta A B
\end{aligned}
$$

In this case the representation is commutative and $A$ is a function of $B$.

In the infinite dimensional case there exist representations where they act as scalar matrices (TASEP, Karimipour Model), assuming that they are scalar we get the following relations:

$$
\begin{array}{r}
\left(\delta_{a a}^{1}+\delta_{a a}^{2}\right) A^{2}-\gamma_{b a} B A-\gamma_{a b} A B=0 \\
-\delta_{a a}^{1} A^{2}+\left(\delta_{b a}+\gamma_{b a}\right) B A-\lambda_{a b} A B-\gamma_{b b}^{1} B^{2}=x_{2} A-x_{1} B \\
-\delta_{a a}^{2} A^{2}+\left(\gamma_{a b}+\delta_{a b}+\lambda_{a b}\right) A B-\gamma_{b b}^{2} B^{2}=x_{1} B-x_{2} A \\
-\delta_{b a} B A-\delta_{a b} A B+\left(\gamma_{b b}^{1}+\gamma_{b b}^{2}\right) B^{2}=0 \\
\lambda_{a} A E=x_{1} E-x_{3} A \\
\lambda_{b} B E=x_{2} E-x_{3} B
\end{array}
$$

One of the equations dependent and we get the following diffusion algebra:

$$
\begin{gathered}
A B\left(\lambda_{a b}+\frac{\delta_{a a}^{1} \gamma_{a b}}{\delta_{a a}^{1}+\delta_{a a}^{2}}+\frac{\gamma_{b b}^{1} \delta_{a b}}{\gamma_{b b}^{1}+\gamma_{b b}^{2}}\right)-B A\left(\frac{\delta_{a a}^{2} \gamma_{b a}}{\delta_{a a}^{1}+\delta_{a a}^{2}}+\frac{\delta_{b a} \gamma_{b b}^{2}}{\gamma_{b b}^{1}+\gamma_{b b}^{2}}\right)=x_{1} B-x_{2} A \\
A^{2}=\frac{\gamma_{b a}}{\delta_{a a}^{1}+\delta_{a a}^{2}} B A+\frac{\gamma_{a b}}{\delta_{a a}^{1}+\delta_{a a}^{2}} A B, \quad B^{2}=\frac{\delta_{b a}}{\gamma_{b b}^{1}+\gamma_{b b}^{2}} B A+\frac{\delta_{a b}}{\gamma_{b b}^{1}+\gamma_{b b}^{2}} A B \\
\lambda_{a} A E=x_{1} E-x_{3} A, \quad \lambda_{b} B E=x_{2} E-x_{3} B
\end{gathered}
$$

Assume that $A$ and $B$ are invertible matrices, then we get the following relations between the inverse matrices:

$$
\begin{aligned}
& 1=\frac{\gamma_{b a}}{\delta_{a a}^{1}+\delta_{a a}^{2}} A^{-1} B+\frac{\gamma_{a b}}{\delta_{a a}^{1}+\delta_{a a}^{2}} B A^{-1} \\
& 1=\frac{\delta_{b a}}{\gamma_{b b}^{1}+\gamma_{b b}^{2}} A B^{-1}+\frac{\delta_{a b}}{\gamma_{b b}^{1}+\gamma_{b b}^{2}} B^{-1} A
\end{aligned}
$$

Denote $K=A^{-1} B$ and $D=B A^{-1}$. Then it follows that $K$ and $D$ satisfy quadratic polynomials:

$$
\begin{aligned}
& \frac{\gamma_{b a}}{\delta_{a a}^{1}+\delta_{a a}^{2}} K^{2}+\left(\frac{\gamma_{a b} \delta_{a b}-\gamma_{b a} \delta_{b a}}{\left(\delta_{a a}^{1}+\delta_{a a}^{2}\right)\left(\gamma_{b b}^{1}+\gamma_{b b}^{2}\right)}-1\right) K+\frac{\delta_{b a}}{\gamma_{b b}^{1}+\gamma_{b b}^{2}}=0 \\
& \frac{\gamma_{a b}}{\delta_{a a}^{1}+\delta_{a a}^{2}} D^{2}+\left(\frac{\gamma_{b a} \delta_{b a}-\gamma_{a b} \delta_{a b}}{\left(\delta_{a a}^{1}+\delta_{a a}^{2}\right)\left(\gamma_{b b}^{1}+\gamma_{b b}^{2}\right)}-1\right) D+\frac{\delta_{a b}}{\gamma_{b b}^{1}+\gamma_{b b}^{2}}=0
\end{aligned}
$$

$\mathrm{RR} \mathrm{n}^{\circ} 7278$ 
Let $K$ and $D$ be two dimensional matrices, then their characteristic polynomials takes zero values at them. And therefore:

$$
\begin{aligned}
K_{11} K_{22}-K_{12} K_{21} & =\frac{\delta_{b a}\left(\delta_{a a}^{1}+\delta_{a a}^{2}\right)}{\gamma_{b a}\left(\gamma_{b b}^{1}+\gamma_{b b}^{2}\right)}, \\
K_{11}+K_{22} & =\frac{\delta_{a a}^{1}+\delta_{a a}^{2}}{\gamma_{b a}}\left(1-\frac{\gamma_{a b} \delta_{a b}-\gamma_{b a} \delta_{b a}}{\left(\delta_{a a}^{1}+\delta_{a a}^{2}\right)\left(\gamma_{b b}^{1}+\gamma_{b b}^{2}\right)}\right), \\
D_{11} D_{22}-D_{12} D_{21} & =\frac{\delta_{a b}\left(\delta_{a a}^{1}+\delta_{a a}^{2}\right)}{\gamma_{a b}\left(\gamma_{b b}^{1}+\gamma_{b b}^{2}\right)}, \\
D_{11}+D_{22} & =\frac{\delta_{a a}^{1}+\delta_{a a}^{2}}{\gamma_{a b}}\left(1+\frac{\gamma_{a b} \delta_{a b}-\gamma_{b a} \delta_{b a}}{\left(\delta_{a a}^{1}+\delta_{a a}^{2}\right)\left(\gamma_{b b}^{1}+\gamma_{b b}^{2}\right)}\right) .
\end{aligned}
$$

Thus there is a wast family of the two dimensional matrices satisfying the quadratic relations. One construct as well the infinite dimensional matrices as block-diagonal. Between $K$ and $A$ we have $q$-commutation relation:

$A K\left(\lambda_{a b}+\frac{\delta_{a a}^{1} \gamma_{a b}}{\delta_{a a}^{1}+\delta_{a a}^{2}}+\frac{\gamma_{b b}^{1} \delta_{a b}}{\gamma_{b b}^{1}+\gamma_{b b}^{2}}\right)-K A\left(\frac{\delta_{a a}^{2} \gamma_{b a}}{\delta_{a a}^{1}+\delta_{a a}^{2}}+\frac{\delta_{b a} \gamma_{b b}^{2}}{\gamma_{b b}^{1}+\gamma_{b b}^{2}}\right)=x_{1} K-x_{2}$

Let us investigate the problem of constructing a two-dimensional matrix $A$ once we have a matrix $K$ given:

$$
A K-q K A=y_{1} K-y_{2},
$$

where

$$
\begin{gathered}
q=\left(\frac{\delta_{a a}^{2} \gamma_{b a}}{\delta_{a a}^{1}+\delta_{a a}^{2}}+\frac{\delta_{b a} \gamma_{b b}^{2}}{\gamma_{b b}^{1}+\gamma_{b b}^{2}}\right) /\left(\lambda_{a b}+\frac{\delta_{a a}^{1} \gamma_{a b}}{\delta_{a a}^{1}+\delta_{a a}^{2}}+\frac{\gamma_{b b}^{1} \delta_{a b}}{\gamma_{b b}^{1}+\gamma_{b b}^{2}}\right) \\
y_{i}=x_{i} /\left(\lambda_{a b}+\frac{\delta_{a a}^{1} \gamma_{a b}}{\delta_{a a}^{1}+\delta_{a a}^{2}}+\frac{\gamma_{b b}^{1} \delta_{a b}}{\gamma_{b b}^{1}+\gamma_{b b}^{2}}\right)
\end{gathered}
$$

Assume $q \neq 1$ and by the shift of variables

$$
A \rightarrow-y_{2} \tilde{A}+\frac{y_{1}}{1-q}
$$

we come to a problem of construction of two $q$-commuting matrices

$$
\tilde{A} K-q K \tilde{A}=1
$$

First consider the case when $A$ is diagonal then we have:

$$
\tilde{A}=\frac{1}{k_{1}(1-q)}\left(\begin{array}{cc}
1 & 0 \\
0 & q^{-1}
\end{array}\right) \quad K=\left(\begin{array}{cc}
k_{1} & k_{2} \\
0 & q k_{1}
\end{array}\right)
$$

$k_{1}$ and $k_{2}$ satisfy a system of equations:

$$
k_{1} k_{2}=\frac{\delta_{b a}\left(\delta_{a a}^{1}+\delta_{a a}^{2}\right)}{\gamma_{b a}\left(\gamma_{b b}^{1}+\gamma_{b b}^{2}\right)}, \quad k_{1}+k_{2}=\frac{\delta_{a a}^{1}+\delta_{a a}^{2}}{\gamma_{b a}}\left(1-\frac{\gamma_{a b} \delta_{a b}-\gamma_{b a} \delta_{b a}}{\left(\delta_{a a}^{1}+\delta_{a a}^{2}\right)\left(\gamma_{b b}^{1}+\gamma_{b b}^{2}\right)}\right)
$$


Matrices $A$ and $B$ explicitly have the following form:

$$
\begin{aligned}
A & =\frac{1}{1-q}\left(\begin{array}{cc}
y_{1} k_{1}-\frac{y_{2}}{y_{1}} & 0 \\
0 & y_{1}-\frac{y_{2}}{q k_{1}}
\end{array}\right), \\
B & =\frac{1}{1-q}\left(\begin{array}{cc}
y_{1} k_{1}-y_{2} & y_{1} k_{2}-\frac{y_{2} k_{2}}{k_{1}} \\
0 & q y_{1} k_{1}-y_{2}
\end{array}\right) .
\end{aligned}
$$

The matrix $D=B A^{-1}$ allows to fix some relations between the rates. It has the following form:

$$
D=\left(\begin{array}{cc}
\frac{y_{1}^{2} k_{1}-y_{1} y_{2}}{y_{1}^{2} k_{1}-y_{2}} & \frac{q k_{1} k_{2}-q y_{2} k_{2}}{q y_{1} k_{1}-y_{2}} \\
0 & q k_{1}
\end{array}\right)
$$

and the relations are the following:

$$
\begin{aligned}
q k_{1} \frac{y_{1}^{2} k_{1}-y_{1} y_{2}}{y_{1}^{2} k_{1}-y_{2}} & =\frac{\delta_{a b}\left(\delta_{a a}^{1}+\delta_{a a}^{2}\right)}{\gamma_{a b}\left(\gamma_{b b}^{1}+\gamma_{b b}^{2}\right)}, \\
\frac{y_{1}^{2} k_{1}-y_{1} y_{2}}{y_{1}^{2} k_{1}-y_{2}}+q k_{1} & =\frac{\delta_{a a}^{1}+\delta_{a a}^{2}}{\gamma_{a b}}\left(1+\frac{\gamma_{a b} \delta_{a b}-\gamma_{b a} \delta_{b a}}{\left(\delta_{a a}^{1}+\delta_{a a}^{2}\right)\left(\gamma_{b b}^{1}+\gamma_{b b}^{2}\right)}\right)
\end{aligned}
$$

we take

$$
|V\rangle=\left(\begin{array}{l}
1 \\
0
\end{array}\right)
$$

and we get the following relations as well

$$
\begin{aligned}
-\frac{x_{1}}{\alpha^{\prime}} & =\frac{1}{1-q}\left(y_{1} k_{1}-\frac{y_{2}}{y_{1}}\right) \\
-\frac{x_{2}}{\beta^{\prime}} & =\frac{1}{1-q}\left(y_{1} k_{1}-y_{2}\right)
\end{aligned}
$$

\section{Tandem queues with stochastic service rates}

\subsection{Model definition}

As already mentioned in Section 2.3, in some cases, on the ring geometry, exclusion processes can be mapped onto a queuing process. These have a fixed number of queues, organized in tandem and in each queue, both the number of clients and the service rate are stochastic, the service being not necessarily a function of the number clients. In some case, each queue taken in isolation may not be a reversible process, with some hysteresis feature relevant to traffic. This family of models is defined more formally as follows: let $\mathcal{G}=(\mathcal{N}, \mathcal{L})$ a network of queues with dynamical (stochastic) service rates. By dynamical service rates, we mean that each single queue $i \in \mathcal{N}$ is represented by a vector $z_{i}(t)=\left(n_{i}(t), \mu_{i}(t)\right) \in E_{i} \subset \mathbb{N} \times \mathbb{R}^{+} . n_{i}(t)$ is the number of clients and $\mu_{i}(t)$ is a service rate, which represents the global transition rate from $z_{i}$ to $z_{i}^{\prime}=\left(n_{i}-1, \mu_{i}^{\prime}\right) \in V_{i}^{-}\left(z_{i}\right)\left(V_{i}^{-}(z)\right.$ is the set of points in $E_{i}$ having one client 
less than $z$ ). From the viewpoint of the statistical physics this process is a generalization of the zero-range process, obtained by adding internal dynamics.

Two sets of transition probability matrices $p_{i}^{ \pm}\left(z, z^{\prime}\right)$ and one set of transition rates $q_{i}^{0}\left(z, z^{\prime}\right)$ are introduced to complete the definition of the process. When a client get served in queue $i$, the state of the departure queue $z_{i}$ is modified according to the set $p_{i}^{-}\left(z, z^{\prime}\right)$ (with $z^{\prime} \in V^{-}(z)$ ) and the state $z_{i+1}$ of the destination queue is modified according the set $p_{i+1}^{+}\left(z, z^{\prime}\right), z^{\prime} \in V^{+}(z)$. We have the normalizations,

$$
\sum_{z^{\prime} \in z} p_{i}^{ \pm}\left(z, z^{\prime}\right)=1, \quad \forall z^{\prime} \in V^{ \pm}(z)
$$

Additional internal transitions are allowed, where the service rate $\mu_{i}$ of queue $i$ changes independently of any arrival or departure. The intensities of these transitions are given by the set $q_{i}^{0}\left(z, z^{\prime}\right), z^{\prime} \in V_{i}^{0}(z)$ of transition rates $\left(V_{i}^{0}(z)\right.$ is the set of points in $E_{i}$ having the same number of clients as $z$ ). The combined set of transition rates,

$q_{i}\left(z, z^{\prime}\right) \stackrel{\text { def }}{=} \lambda p_{i}^{+}\left(z, z^{\prime}\right) \mathbb{1}_{\left\{z^{\prime} \in V_{i}^{+}(z)\right\}}+\mu(z) p_{i}^{-}\left(z, z^{\prime}\right) \mathbb{1}_{\left\{z^{\prime} \in V_{i}^{-}(z)\right\}}+q_{i}^{0}\left(z, z^{\prime}\right) \mathbb{1}_{\left\{z^{\prime} \in V_{i}^{0}(z)\right\}}$,

defines for each $i \in \mathcal{N}$ a continuous time Markov process representing the dynamics of each queue taken in isolation. The model is then entirely specified by the state graph of the single queue which set of nodes $\{(n, \mu)\}$ is a subset of $\mathbb{N} \times \mathbb{R}_{+}$, and in which each transition is represented by an oriented edge (see e.g. Figures 2 and 3).

In the following, we will assume that the network under consideration is ergodic and admits a unique stationary measure.

\subsection{Product form of clusters at steady-state}

In the stationary regime, the invariant measure of a network of reversible queues is known to factorize into a product form in general [18], which allows to compute many equilibrium quantities explicitly. With a dynamical rate it is interesting to check for such properties and in this section we study the conditions under which the stationary state has a product form. We can establish the following sufficient conditions:

Theorem 4.1. Let $\pi_{i}^{\lambda}$ denotes the steady state probability corresponding to queue $i$ taken in isolation and fed with a Poisson process with rate $\lambda$. If the following partial balance equations are satisfied,

$$
\begin{gathered}
\sum_{z \in V^{+}\left(z_{i}\right)} \mu(z) p_{i}^{-}\left(z, z_{i}\right) \pi_{i}^{\lambda}(z)=\lambda \pi_{i}^{\lambda}\left(z_{i}\right), \\
\mu\left(z_{i}\right) \pi_{i}^{\lambda}\left(z_{i}\right)+\sum_{z \in V_{i}^{0}\left(z_{i}\right)} q_{i}^{0}\left(z_{i}, z\right) \pi_{i}^{\lambda}\left(z_{i}\right)= \\
\sum_{z \in V^{-}\left(z_{i}\right)} \lambda p^{+}\left(z, z_{i}\right) \pi_{i}^{\lambda}(z)+\sum_{z \in V_{i}^{0}\left(z_{i}\right)} q_{i}^{0}\left(z, z_{i}\right) \pi_{i}^{\lambda}(z),
\end{gathered}
$$

then the joint probability measure of the network has the following product form at steady state:

$$
P\left(S=\left\{z_{i}, i \in \mathcal{N}\right\}\right)=\frac{\prod_{i \in \mathcal{N}} \pi_{i}^{\lambda}\left(z_{i}\right)}{P\left(\sum_{i} n_{i}=N\right)}
$$


Proof. Using (18), the global balance equations reads

$$
\begin{aligned}
\sum_{i \in \mathcal{N}}\left[\sum_{\substack{z^{\prime} \in V^{+}\left(z_{i}\right) \\
z^{\prime \prime} \in V^{-}\left(z_{i+1}\right)}} \mu\left(z^{\prime}\right) p^{-}\left(z^{\prime}, z_{i}\right) p^{+}\left(z^{\prime \prime}, z_{i+1}\right) \frac{\pi_{i}^{\lambda}\left(z^{\prime}\right) \pi_{i+1}^{\lambda}\left(z^{\prime \prime}\right)}{\pi_{i}^{\lambda}\left(z_{i}\right) \pi_{i+1}^{\lambda}\left(z_{i+1}\right)}\right. \\
\left.+\sum_{z \in V_{i}^{0}\left(z_{i}\right)} q_{i}^{0}\left(z, z_{i}\right) \frac{\pi_{i}^{\lambda}(z)}{\pi_{i}^{\lambda}\left(z_{i}\right)}\right] P\left(S=\left\{z_{i}\right\}\right) \\
=\sum_{i \in \mathcal{N}}\left[\mu\left(z_{i}\right)+\sum_{z \in V_{i}^{0}\left(z_{i}\right)} q_{i}^{0}\left(z_{i}, z\right)\right] P\left(S=\left\{z_{i}\right\}\right)
\end{aligned}
$$

To find a sufficient condition, let us use (21), and select term $i$ in the first term of the left hand side and term $i+1$ otherwise in the preceding equation, yielding $\forall i \in \mathcal{N}$,

$$
\begin{aligned}
& \sum_{\substack{z^{\prime} \in V^{+}\left(z_{i}\right) \\
z^{\prime \prime} \in V^{-}\left(z_{i+1}\right)}} \mu\left(z^{\prime}\right) p^{-}\left(z^{\prime}, z_{i}\right) p^{+}\left(z^{\prime \prime}, z_{i+1}\right) \pi_{i}^{\lambda}\left(z^{\prime}\right) \pi_{i+1}^{\lambda}\left(z^{\prime \prime}\right)+ \\
& \sum_{z \in V^{0}\left(z_{i+1}\right)} q_{i+1}^{0}\left(z, z_{i+1}\right) \pi_{i}^{\lambda}\left(z_{i}\right) \pi_{i+1}^{\lambda}(z) \\
& =\mu\left(z_{i+1}\right) \pi_{i}^{\lambda}\left(z_{i}\right) \pi_{i+1}^{\lambda}\left(z_{i+1}\right)+\sum_{z \in V^{0}\left(z_{i+1}\right)} q_{i+1}^{0}\left(z_{i+1}, z\right) \pi_{i}^{\lambda}\left(z_{i}\right) \pi_{i+1}^{\lambda}\left(z_{i+1}\right) .
\end{aligned}
$$

After multiplying this last equation by $\lambda$, this sufficient condition rewrites

$$
\begin{gathered}
\left(\sum_{z^{\prime} \in V^{+}\left(z_{i}\right)} \mu\left(z^{\prime}\right) p^{-}\left(z^{\prime}, z_{i}\right) \pi_{i}^{\lambda}\left(z^{\prime}\right)\right) \\
\left(\lambda \sum_{z^{\prime \prime} \in V^{-}\left(z_{i+1}\right)} p^{+}\left(z^{\prime \prime}, z_{i+1}\right) \pi_{i+1}^{\lambda}\left(z^{\prime \prime}\right)\right) \\
=\lambda \pi_{i}^{\lambda}\left(z_{i}\right) \times \\
\left(\mu\left(z_{i+1}\right) \pi_{i+1}^{\lambda}\left(z_{i+1}\right)+\sum_{z \in V^{0}\left(z_{i+1}\right)} q_{i+1}^{0}\left(z_{i+1}, z\right) \pi_{i+1}^{\lambda}\left(z_{i+1}\right)-q_{i+1}^{0}\left(z, z_{i+1}\right) \pi_{i+1}^{\lambda}(z)\right),
\end{gathered}
$$

which is satisfied $\forall i \in \mathcal{N}$ under the two sets of sufficient conditions (19) and (20).

Note that reversible processes are special cases of processes obeying $(19,20)$ and, in this respect, our result is an adaptation to our context of the general results of Kelly concerning product forms in queueing networks [18]. The next obvious question then is whether there exists any such process which is nonreversible.

\subsection{Examples for traffic models}

Let us answer to this by considering two examples. The isolated-queue stategraph corresponding to the first one is sketched in Figure 2(b). Using the labelling $(0)=\left(0, \mu_{0}\right),(1)=\left(1, \mu_{1}\right),(2)=\left(1, \mu_{2}\right),(3)=\left(2, \mu_{4}\right)$ and $(i)=$ 


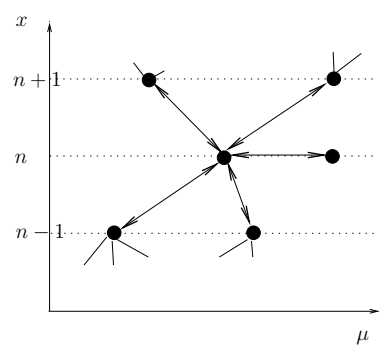

(a)
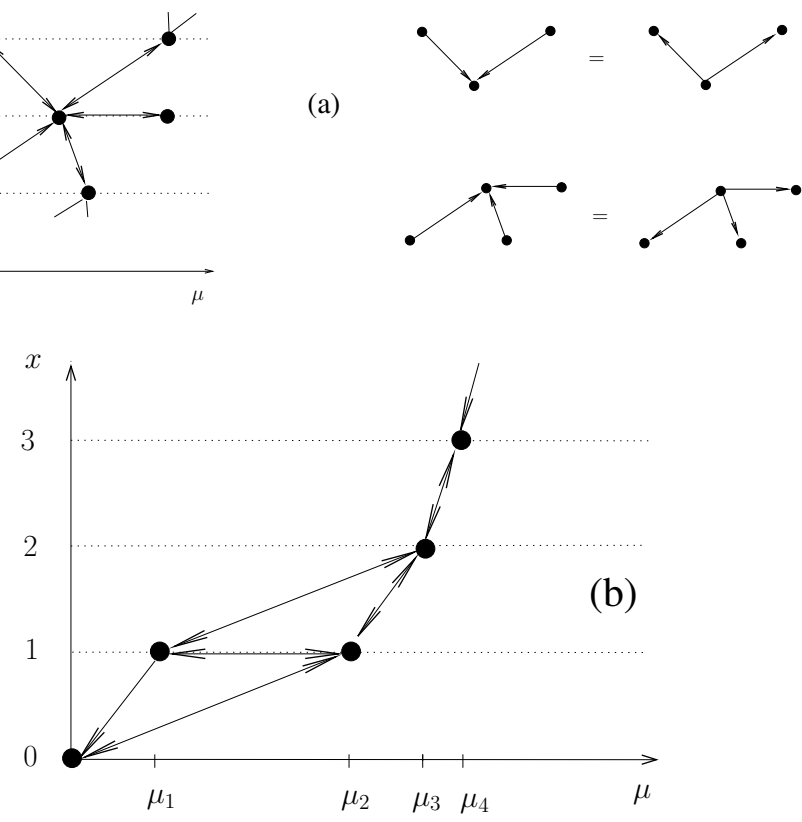

Figure 2: (a) Partial balance condition corresponding to (19) and (20). (b) Example of a non-reversible single queue process obeying to this condition.

$\left(n-2, \mu_{i}\right)$ for $i \geq 4$ for the different states of the isolated queue, these partial balance conditions written for the nodes $(i), i<4$ yield four independent relations between the single queues steady states probabilities $\pi_{i}$ :

$$
\begin{aligned}
\lambda \pi_{0} & =\mu_{1} \pi_{1}+\mu_{2} \pi_{2}, & \lambda \pi_{1}=\mu_{3} p_{31} \pi_{3}, \\
q_{21} \pi_{2} & =\left(q_{12}+\mu_{1}\right) \pi_{1}, & \lambda \pi_{2}=\mu_{3} p_{32} \pi_{3},
\end{aligned}
$$

which are actually compatible iff:

$$
\frac{p_{32}}{p_{31}}=\frac{q_{12}+\mu_{1}}{q_{21}} .
$$

Letting $p \stackrel{\text { def }}{=} p_{31}=1-p_{32}$, this corresponds to

$$
p=\frac{q_{21}}{\mu_{1}+q_{12}+q_{21}} .
$$

When this hold the joint measure of the tandem queue takes the product form (21) whereas this example is by construction never reversible (transitions $0 \rightarrow 1$ is absent), with the single queue invariant measure simply reading

$$
\begin{aligned}
& \pi_{1}=\frac{\lambda q_{12}}{\mu_{1} \mu_{2}+q_{12} \mu_{2}+q_{21} \mu_{1}} \pi_{0}, \quad \pi_{2}=\frac{\lambda\left(\mu_{1}+q_{21}\right)}{\mu_{1} \mu_{2}+q_{12} \mu_{2}+q_{21} \mu_{1}} \pi_{0}, \\
& \pi_{3}=\frac{\lambda^{2}\left(\mu_{1}+q_{12}+q_{21}\right)}{\mu_{3}\left(\mu_{1} \mu_{2}+q_{12} \mu_{2}+q_{21} \mu_{1}\right)} \pi_{0}, \quad \pi_{n \geq 4}=\left[\prod_{i=4}^{n} \frac{\lambda}{\mu_{i}}\right] \frac{\lambda q_{12}}{\mu_{1} \mu_{2}+q_{12} \mu_{2}+q_{21} \mu_{1}} \pi_{0},
\end{aligned}
$$




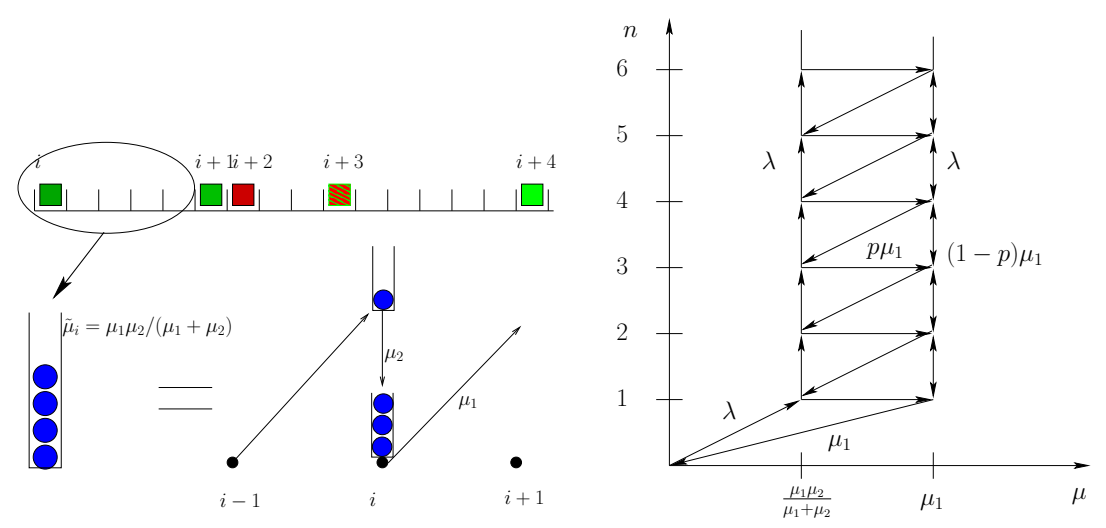

Figure 3: Composite traffic tandem queue process. Equivalence with multispeed ASEP (left). Corresponding process in isolation, $p=\rho_{1}^{n-1} \frac{\rho_{1}-\rho_{2}}{\rho_{1}^{n}-\rho_{2}^{n}}$ (right)

where $\pi_{0}$ is set upon normalization. Our second example is depicted in Figure 3. It represents an exclusion process on a ring, where particles, to hop to the next site have to follow a cascading process. For sake of simplicity let us limit ourselves to a two stage cascade, where empty site are of two different types: either blocking type $B$ and non-blocking type $A$. The transition rules then read:

$$
\begin{array}{ll}
V A \stackrel{\lambda_{a b}}{\longrightarrow} B V & \text { vehicle hopping } \\
V B \stackrel{\gamma_{v b}}{\longrightarrow} V A & \text { blocking site becomes non-blocking } \\
A B \stackrel{\gamma_{a b}}{\longrightarrow} A A & \text { blocking site becomes non-blocking }
\end{array}
$$

The resulting exclusion process, display different type of car motion symmetry breaking mechanism between acceleration/deceleration depending on the free space ahead. This model is mapped on a composite queuing process as follows: cars represent composite queues, with client arriving in the type $B$ (first stage of the composite queue) and able to jump to the second stage in type $A$ when they reach the server of the queue, i.e. when the left neighbor is of type $A$. It has a product form since each single queue taken in isolation is reversible. An hysteresis mechanism for each cycle of deceleration and acceleration takes place due to the non-reversibility of the single queue taken in isolation depicted on Figure 3(b). Indeed this queue is an effective queue equivalent to the composite one and is clearly non-reversible. Nevertheless the partial balance sufficient condition for having a product form are fulfilled. Indeed in the example of the figure the isolated composite queue has for invariant measure

$$
\begin{aligned}
\pi\left(n, \tilde{\mu}=\frac{\mu_{1} \mu_{2}}{\mu_{1}+\mu_{2}}\right) & =\left(1-\rho_{1}\right)\left(1-\rho_{2}\right) \rho_{1}^{n}, \\
\pi\left(n, \tilde{\mu}=\mu_{1}\right) & =\left(1-\rho_{1}\right)\left(1-\rho_{2}\right) \frac{\rho_{1}^{n}-\rho_{2}^{n}}{\rho_{1}-\rho_{2}},
\end{aligned}
$$

with $\rho_{1,2}=\lambda / \mu_{1,2}$. Computing the fluxes yields then the desired partial balance conditions. It seems that this procedure of replacing composite queues 
with effective one yield automatically non-reversible queues fulfilling the partial balance conditions.

The analysis of the fundamental diagram follows then almost straightforwardly when such product form is present, although some non-trivial feature are also expected depending on the value of the spectral gap between the steadystate and the first excited state.

\subsection{Computing the fundamental diagram}

In practice, points plotted in experimental FD studies are obtained by averaging data from static loop detectors over a few minutes (see e.g. [2]). This is difficult to do with our queue-based model, for which a space average is much easier to obtain. The equivalence between time and space averaging is not an obvious assumption, but since jams are moving, space and time correlations are combined in some way [15] and we consider this assumption to be quite safe. In what follows, we will therefore compute the FD by considering either the joint probability measure the conditional probability measure $P_{\lambda}(\phi \mid d)$ for a closed system, where

$$
\left\{\begin{array} { l l } 
{ d = \frac { N } { N + L } , } \\
{ \phi = \frac { \Phi } { N + L } , }
\end{array} \quad \text { with } \left\{\begin{array}{ll}
L=\sum_{i=1}^{L} X_{i} & \text { number of queues } \\
\Phi=\sum_{i=1}^{L} R_{i} \mathbb{1}_{\left\{X_{i}>0\right\}} & \text { integrated flow }
\end{array}\right.\right.
$$

are spatial averaged quantities and represent respectively the density and the traffic flow. The numbers $N$ of vehicles and $L$ of queues are fixed, meaning in the statistical physics parlance, that we are working with the canonical ensemble. As a result this constraints yields the following form of the joint probability measure:

$$
P(S)=\frac{1}{Z_{L}[N]} \prod_{i=1}^{L} P_{\lambda}\left(x_{i}, \mu_{i}\right)
$$

with the canonical partition function

$$
Z_{L}[N] \stackrel{\text { def }}{=} \sum_{\left\{x_{i}\right\}} \prod_{i=1}^{L} P_{\lambda}\left(x_{i}\right) \delta\left(N-\sum_{i=1}^{L} x_{i}\right) .
$$

These expressions are actually independent of $\lambda$ in this specific ring geometry. The density-flow conditional probability distribution takes the form

$$
P(\phi \mid d)=\frac{Z_{L}[N, \Phi]}{Z_{L}[N]}
$$

with

and

$$
N=\frac{d}{1-d} L, \quad \Phi=\frac{\phi}{1-d} L
$$$$
Z_{L}[N, \Phi] \stackrel{\text { def }}{=} \sum_{\left\{x_{i}\right\}} \int \cdots \int \prod_{i=1}^{L} P_{\lambda}\left(x_{i}, d \mu_{i}\right) \delta\left(N-\sum_{i=1}^{L} x_{i}\right) \delta\left(\Phi-\sum_{i=1}^{L} \mu_{i} \mathbb{1}_{\left\{x_{i}>0\right\}}\right) .
$$ 
Note (by simple inspection, see e.g. [18]) that $P(\phi \mid d)$ is independent of $\lambda$.

Let us consider $L_{x, \mu}$ the number of queues having $x$ clients and service rate $\mu$. Assuming $\mu$ runs over discrete values, the partition function (26) can be recast in:

$$
\begin{aligned}
Z_{L}(N, \Phi) & =\sum_{\left\{L_{x, \mu}\right\}} \frac{L !}{\prod_{x, \mu} L_{x, \mu} !} \prod_{x, \mu} p_{\lambda}(x, \mu)^{L_{x, \mu}} \\
& \times \delta\left(L-\sum_{x, \mu} L_{x, \mu}\right) \delta\left(N-\sum_{x, \mu} x L_{x, \mu}\right) \delta\left(\Phi-\sum_{x>0, \mu} \mu L_{x, \mu}\right)
\end{aligned}
$$

Let us introduce the large deviation functional, $\mathcal{F}$, according to

$$
Z_{L}(N, \Phi) \propto \exp (-L \mathcal{F}[y])
$$

with

$$
y(x, \mu) \stackrel{\text { def }}{=} \frac{L_{x, \mu}}{L} .
$$

Keeping only the leading terms in the Stirling formula $\mathcal{F}$ reads

$$
\begin{aligned}
\mathcal{F}(y) & =\sum_{x, \mu} y(x, \mu) \log \frac{y(x, \mu)}{p_{\lambda}(x, \mu)} \\
& +\lambda_{x} \sum_{x, \mu} x y(x, \mu)+\lambda_{y} \sum_{x, \mu} y(x, \mu)+\lambda_{\phi} \sum_{x, \mu} \mu y(x, \mu),
\end{aligned}
$$

where Lagrange multipliers have been introduced as well as to enforce the constraints. The variational principle here amounts to look for a distribution $y$ minimizing the mutual information with the product form while satisfying the constraints, in this sense it is equivalent to the Bethe approximation. The saddle point equation yield

$$
y(x, \mu)=p_{\lambda}(x, \mu) e^{-\lambda_{y}-x \lambda_{x}-\mu \lambda_{\phi}-1},
$$

with the Lagrange multipliers obtained after solving the constraints

$$
\left\{\begin{array}{l}
\sum_{x, \mu} x y(x, \mu)=\frac{d}{1-d}-x_{c} \\
\sum_{x, \mu} y(x, \mu)=1 \\
\sum_{x>0, \mu} \mu y(x, \mu)=\frac{\phi}{1-d} .
\end{array}\right.
$$

Preceding results are recovered upon identifying $\mu^{\star}$ with $\lambda e^{-\lambda_{x}}$ and $y_{\emptyset}=\exp \left(-\lambda_{y}-\right.$ 1). The resulting expression of the free energy at this variational point reads

$$
\mathcal{F}\left(\mu^{*}, \lambda_{\phi}\right)=\left(\frac{d}{1-d}-x_{c}\right) \log \frac{\mu^{\star}}{\mu_{0}}-\lambda_{\phi} \frac{\phi}{1-d}+\log y_{\emptyset},
$$

where $\mu^{*}$ and $\lambda_{\phi}$ implicitly determine the density $\rho$ and the flux $\phi$ so that the fundamental diagram density is proportional to $\exp \left(-\mathcal{F}\left(\mu^{*}, \lambda_{\phi}\right)\right)$ in the $(\rho, \phi)$ quarter plane. 


\section{Conclusion and Perspectives}

This paper is an attempt to focus from many different viewpoints, as far as exact solvability is concerned on the problem of the fundamental diagram of single lane road traffic. As can be seen, steady state properties of the models presented here are quite well understood, either using matrix ansatz in some cases, were new example of diffusion algebra are found in Section 3.4 or with queueing process view point, where condition to have a product form have been extended from the usual reversibility one, in Section 4.2.

The dynamical properties of our models are as expected, much more difficult to account for, as full integrability seems to be associated to oversimplified assumptions, although the integrable family of models found in Section 3.1 seems worth to be investigated further. The traffic models with acceleration/deceleration dynamics feature coagulation/decoagulation dynamics when the vehicles change their speed. With two different speed levels a rich dynamics is already present. The difference from pure coagulation/decoagulation models comes from the hopping dynamics, which prevents particles/vehicles from coagulation. We can think of these model effectively as a statistical physics model of particles in an external driving field. A conjecture would be, that in general, we have three phases: low density/high density and a gap-less phase. These phases depend on relation between the relative hopping rate $\lambda_{a b}$ and the coagulation rate. The difference with solvable models in statistical physics is a strict asymmetry of hopping; would there be a slight diffusion to the left, the model then would not be too far away from simple coagulation/decoagulation model, which is known to be solvable exactly by the empty interval method and by the free fermions for particular choice of the rates. The solution is consistent with a three phases structure of the fundamental diagram. The tool which works as well good for solvable or not solvable models is the Matrix Product Ansatz(MPA). When a model turns out to be integrable then MPA allows generalization to the dynamic MPA (DMPA), which turns out to be equivalent to the Bethe Ansatz.

Further work could be devoted to analyze in more details near equilibrium properties, by looking e.g. more closely at the behavior of the spectral gap and the density waves perturbing the stationary state, using exact methods developed in Section 3 or approximate ones. Additionally, the models considered here are limited to single lane traffic and could be easily generalized to multilane, by e.g. coupling exclusion processes. Looking for $R$ matrices associated to such kind of models could be interesting.

Acknowledgments This work was supported by the French National Research Agency (ANR) grant No ANR-08-SYSC-017.

\section{References}

[1] M. Schreckenberg, A. Schadschneider, K. Nagel, and N. Ito. Discrete stochastic models for traffic flow. Phys. Rev., E51:2339, 1995.

[2] B.S. Kerner. The Physics of Traffic. Springer Verlag, 2005.

[3] M. Schönhof and D. Helbing. Criticism of three-phase traffic theory. Transportation Research, 43:784-797, 2009. 
[4] R.J. Baxter. Exactly Solved Models in Statistical Physics. London: Academic Press, 1982.

[5] K. Nagel and M. Schreckenberg. A cellular automaton model for freeway traffic. J. Phys. I,2, pages 2221-2229, 1992.

[6] R. Barlović, L. Santen, A. Schadschneider, and M. Schreckenberg. Metastable states in cellular automata for traffic flow. Eur. Phys. J., B5:793, 1998.

[7] Y. Sugiyama et al. Traffic jams without bottlenecks: experimental evidence for the physical mechanism of the formation of a jam. New Journal of Physics, 10:1-7, 2008 .

[8] B. Derrida, M. R. Evans, V. Hakim, and V. Pasquier. Exact solution for 1d asymmetric exclusion model using a matrix formulation. J. Phys. A: Math. Gen., 26:1493-1517, 1993.

[9] R.A. Blythe and M.R. Evans. Nonequilibrium steady states of matrix-product forms: a solver's guide. J.Phys. A: Math. Theor., 40:R333-R441, 2007.

[10] T. M. Liggett. Interacting Particle Systems. Springer, Berlin, 2005.

[11] F. Spitzer. Interaction of markov processes. Adv. Math., 5:246, 1970

[12] L. Cantini. Algebraic bethe ansatz for the two species asep with different hopping rates. J. Phys. A: Math. Theor., 41:095001, 2008.

[13] V. Karimipour. A multi-species asep and its relation to traffic flow. Phys. Rev., E59:205, 1999.

[14] C. Furtlehner and J.M. Lasgouttes. A queueing theory approach for a multi-speed exclusion process. In Traffic and Granular Flow' '07, pages 129-138, 2007.

[15] K. Nagel and M. Paczuski. Emergent traffic jams. Phys. Rev. E, 51(4):2909-2918, 1995.

[16] D. ben Avraham and S. Havlin. Diffusion and Reactions in Fractals and Disordered Systems. Cambridge University Press, 2000.

[17] S. Sandow H.Hinrichsen and I. Peschel. On matrix product ground states for reaction - diffusion models. J. Phys. A: Math. Gen., 29:2643, 1996.

[18] F. P. Kelly. Reversibility and stochastic networks. John Wiley \& Sons Ltd., 1979. Wiley Series in Probability and Mathematical Statistics. 
Centre de recherche INRIA Saclay - Île-de-France

Parc Orsay Université - ZAC des Vignes

4, rue Jacques Monod - 91893 Orsay Cedex (France)

Centre de recherche INRIA Bordeaux - Sud Ouest : Domaine Universitaire - 351, cours de la Libération - 33405 Talence Cedex Centre de recherche INRIA Grenoble - Rhône-Alpes : 655, avenue de l'Europe - 38334 Montbonnot Saint-Ismier

Centre de recherche INRIA Lille - Nord Europe : Parc Scientifique de la Haute Borne - 40, avenue Halley - 59650 Villeneuve d'Ascq Centre de recherche INRIA Nancy - Grand Est : LORIA, Technopôle de Nancy-Brabois - Campus scientifique 615, rue du Jardin Botanique - BP 101 - 54602 Villers-lès-Nancy Cedex

Centre de recherche INRIA Paris - Rocquencourt : Domaine de Voluceau - Rocquencourt - BP 105 - 78153 Le Chesnay Cedex

Centre de recherche INRIA Rennes - Bretagne Atlantique : IRISA, Campus universitaire de Beaulieu - 35042 Rennes Cedex

Centre de recherche INRIA Sophia Antipolis - Méditerranée : 2004, route des Lucioles - BP 93 - 06902 Sophia Antipolis Cedex 